\title{
Direct Tip-Sample Force Estimation for High-Speed Dynamic Mode Atomic Force Microscopy
}

\author{
Kai S. Karvinen, Student Member, IEEE, Michael G. Ruppert, Student Member, IEEE, Kaushik Mahata, \\ and S. O. R. Moheimani, Fellow, IEEE
}

\begin{abstract}
We present new insights into the modeling of the microcantilever in dynamic mode atomic force microscopy and outline a novel high-bandwidth tip-sample force estimation technique for the development of high-bandwidth $z$-axis control. Fundamental to the proposed technique is the assumption that in tapping mode atomic force microscopy, the tip-sample force takes the form of an impulse train. Formulating the estimation problem as a Kalman filter, the tip-sample force is estimated directly; thus, potentially enabling high-bandwidth $z$-axis control by eliminating the dependence of the control technique on microcantilever dynamics and the amplitude demodulation technique. Application of this technique requires accurate knowledge of the models of the microcantilever; a novel identification method is proposed. Experimental data are used in an offline analysis for verification.
\end{abstract}

Index Terms-Atomic force microscopy, dynamic mode, Kalman filter, microcantilever, tip-sample force, $z$-axis control.

\section{INTRODUCTION}

$\mathbf{T}$ HE introduction of the atomic force microscope (AFM) in 1986 [1] enabled unrivaled levels of performance in microscopy. For the past 25 years, the AFM has been integral in the investigation of the nanoscale.

Tapping mode atomic force microscopy [2], [3] is possibly the most well-known AFM technique. Despite its suitability for the analysis of a range of samples, one limitation of tapping mode AFM is its low imaging bandwidth owing primarily to the resonant dynamics of the microcantilever and the amplitude estimation method [4]. Since direct measurement of the tip-sample force is not possible, the $z$-axis feedback controller regulates the demodulated amplitude. While improvements have been made to the $z$-axis feedback loop, these typically focus on the replacement of the PI controller with advanced model-based controllers [5]-[7]. The application of advanced $Q$ control techniques [8][10] and high-bandwidth amplitude demodulation techniques [11] can also improve the imaging performance, but ultimately the transient response of the cantilever and demodulation technique still impose a limit.

Manuscript received February 9, 2014; accepted September 25, 2014. Date of publication September 30, 2014; date of current version November 6, 2014. The review of this paper was arranged by Associate Editor L. Dong.

K. S. Karvinen is with the The University of Newcastle, Callaghan, NSW 2308, Australia (e-mail: kai.karvinen@uon.edu.au).

M. G. Ruppert, K. Mahata, and S. O. R. Moheimani are with the School of Electrical Engineering and Computer Science, University of Newcastle, Callaghan, NSW 2308, Australia (e-mail: michael.ruppert@uon.edu.au; Kaushik.Mahata@newcastle.edu.au; reza.moheimani@newcastle.edu.au).

Color versions of one or more of the figures in this paper are available online at http://ieeexplore.ieee.org.

Digital Object Identifier 10.1109/TNANO.2014.2360878
Until now, tapping mode AFM has generally not been associated with high-speed imaging. However, recent results highlighting the observation of dynamic biological processes at the nanoscale provide a strong motivation to develop real-time dynamic mode AFM [12]-[14]. Currently available commercial high-speed AFM systems typically perform imaging in liquids using very small cantilevers with low force constants and very low $Q$ factors; no high-speed techniques currently operate in air with standard cantilevers. In general, many of these existing high-speed AFM systems utilize heavily optimized setups, but fundamentally operate within the framework of conventional amplitude modulation AFM.

In this contribution, we present new insights into the modeling of the microcantilever in dynamic mode atomic force microscopy from a systems perspective. We justify the selection of more sophisticated models, and using these results, we propose a direct tip-sample force estimation technique, which potentially enables tip-sample force regulation leading to significant improvements in the $z$-axis bandwidth. The tip-sample force estimation problem is formulated using the Kalman filter framework. To demonstrate application of this technique, we propose an experiment to accurately characterize and validate the microcantilever models and conclude by verifying the technique with measured data obtained from an AFM scan. The proposed technique is developed with the intention to completely bypass conventional tapping mode AFM operation and to enable highbandwidth imaging in air.

\section{OVERVIEW OF $z$-AXIS CONTROL}

\section{A. Tapping Mode Atomic Force Microscopy}

In tapping mode AFM, typically one of the flexural modes of a microcantilever is excited as it is brought into close proximity with a sample. Modern AFMs commonly utilize a laser and photodiode to estimate the tip displacement. Conventional $z$-axis control schemes then utilize amplitude demodulation and PI control to regulate the oscillation amplitude of the microcantilever through actuation of the $z$-axis nanopositioner. Limitations of the imaging bandwidth are primarily the result of the transient response of the microcantilever and the time delay of the amplitude demodulation technique. The diagram in Fig. 1 highlights the operation of tapping mode AFM.

\section{B. Advanced z-Axis Control for Dynamic Mode AFM}

Initial efforts to improve the imaging bandwidth, without modifying the hardware, focused on the design of advanced controllers to replace the PI controller. Both $H_{\infty}$ loop 


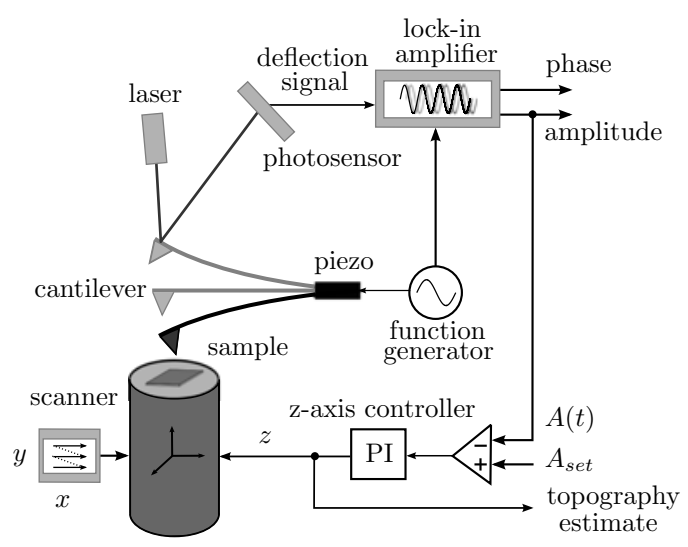

Fig. 1. Schematic overview of tapping mode atomic force microscopy highlighting the $z$-axis feedback loop.

shaping and $H_{\infty}$ stacked sensitivity techniques have been demonstrated in dynamic mode AFM [5], [6], and highbandwidth $H_{\infty}$ controllers have been implemented using a field programmable analog array [7]. Repetitive controllers have also been demonstrated [15]. While these model-based controllers perform better than the standard PI controller, their design requires an intimate knowledge of control theory, which cannot be expected from most AFM practitioners. Furthermore, they are all cast within the framework of amplitude modulation atomic force microscopy, where there are strict limits on the achievable imaging bandwidth. Overcoming these limitations involves the development of novel control techniques, which eliminate any dependence on the microcantilever dynamics or demodulation techniques.

An example of such a technique focuses on the estimation and control of the tip-sample force [16]. A closed-loop observer was utilized to estimate the cantilever tip displacement, velocity, and tip-sample force. The microcantilever was modeled as a simple mass-spring-damper system, and the tip-sample force was modeled as an impulse whose magnitude can be approximated as $-2 m v_{\text {in }}-m a_{e} \pi \sqrt{m / k}$, where $k$ is the spring constant, $m a_{e}$ is the tip-sample force, and $v_{\text {in }}$ is the incident velocity of the cantilever on the sample. The authors presented computer simulations to verify the concept and also highlighted the independence of the control technique from the transient response of the microcantilever. However, no experimental demonstration of this technique has been reported.

The transient force technique is another method which does not fit within the conventional AM-AFM framework, instead utilizing a likelihood test and an observer to determine when tip-sample contact is established [17], [18]. This technique also assumes that the tip-sample force is an impulse and its highbandwidth performance has been experimentally verified. However, it does not directly estimate the tip-sample force.

\section{New Insights Into System Modeling}

The nonlinear tip-sample interaction in dynamic mode atomic force microscopy is often modeled as a linear system with a nonlinear feedback element [19]-[21] as shown in Fig. 2(a).
This model has been effective in describing complex cantilever dynamics.

We propose an expansion of this model and primarily seek to differentiate between two cantilever transfer functions which describe the cantilever system, namely $G_{\text {in }}(s)$ and $G_{\text {tip }}(s)$. The LTI system $G_{\text {in }}(s)$ describes the displacement $y$ resulting from the input actuation $u$, and the LTI system $G_{\text {tip }}(s)$ describes the displacement $y$ resulting from the tip-sample force interaction $w$. Clearly, since these two transfer functions describe the same physical system, the poles will be identical. However, since they represent different input-output relationships, the zeros will be different. Fig. 2(b) highlights the two-input single-output model of the cantilever. It is important to distinguish between these systems in describing the dynamics of the microcantilever in dynamic mode atomic force microscopy. When a piezoelectric actuator is bonded to the microcantilever, the transfer functions of the first $N$ modes take the following form [23]

$$
\begin{aligned}
G_{\text {in }}(s) & =\sum_{i=1}^{N} \frac{\kappa_{i} \omega_{i}^{2}}{s^{2}+2 \zeta_{i} \omega_{i} s+\omega_{i}^{2}}+D_{1} \\
G_{\text {tip }}(s) & =\sum_{i=1}^{N} \frac{\gamma_{i} \omega_{i}^{2}}{s^{2}+2 \zeta_{i} \omega_{i} s+\omega_{i}^{2}}+D_{2}, \gamma_{i}>0
\end{aligned}
$$

where $\zeta_{i}$ and $\omega_{i}$ represent the damping ratio and the resonance frequency for the $i$ th mode. The constraint $\gamma_{i}>0$ in (2) ensures that $G_{\text {tip }}(s)$ is collocated, which is due to sensing and actuation occurring at the same physical point on the microcantilever. To incorporate the effect of truncated modes, the transfer functions can be supplemented with feedthrough terms $D_{1}$ and $D_{2}$ [22].

Augmenting this microcantilever model into the conventional model, our proposed model of the microcantilever in dynamic mode atomic force microscopy is highlighted in Fig. 2(c).

\section{Proposed $z$-Axis Control Scheme}

\section{A. Estimation of the Tip-Sample Force $F_{\mathrm{ts}}$}

Consider the $z$-axis control schemes in Fig. 3. While the tipsample force function $\Phi(y, z)$ and the instantaneous tip-sample force $F_{\mathrm{ts}}(t)$ are not easy to determine, the actuation voltage $u$ and the microcantilever displacement $y$ are easily measured. Furthermore, it is possible to characterize the linear systems $G_{\text {in }}(s)$ and $G_{\text {tip }}(s)$. Consider then that

$$
y(t)=\left(g_{\text {in }} * u\right)(t)+\left(g_{\text {tip }} * F_{\text {ts }}\right)(t)
$$

where $*$ is the convolution operator. Let us define $\psi(t)$ as

$$
\psi(t)=\left(g_{\mathrm{tip}} * F_{\mathrm{ts}}\right)(t)=y(t)-\left(g_{\mathrm{in}} * u\right)(t)
$$

where $y(t), u(t)$, and $g_{\text {in }}(t)$ and $g_{\text {tip }}(t)$ represent the microcantilever tip displacement, the actuation signal, and the microcantilever impulse response functions, respectively. We assume that an accurate model $G_{\text {tip }}(s)$ is available and since $g_{\text {tip }}(t)$ decays exponentially, we can find $T_{d}$ such that

$$
g_{\text {tip }}(t)=0, \quad \text { if } \quad t<0 \quad \text { or } t>T_{d}
$$




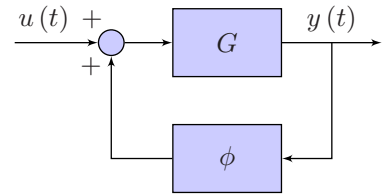

(a)

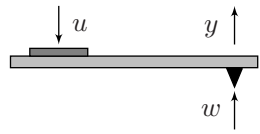

(b)

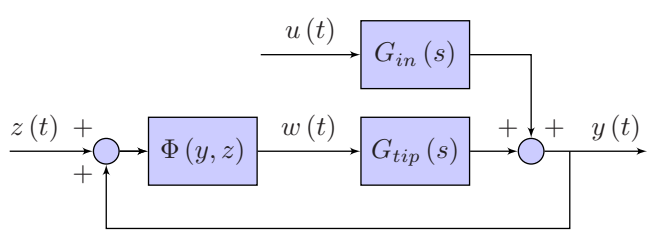

(c)

Fig. 2. (a) Conventional approach to modeling the nonlinear tip-sample interaction in dynamic mode AFM. (b) Two-input single-output model of the piezoelectrically actuated microcantilever. (c) Proposed approach to modeling the nonlinear tip-sample interaction in dynamic mode AFM.

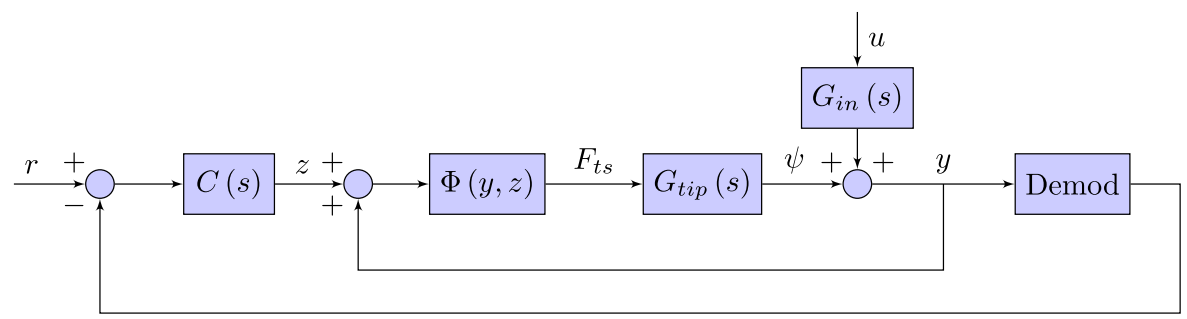

(a)

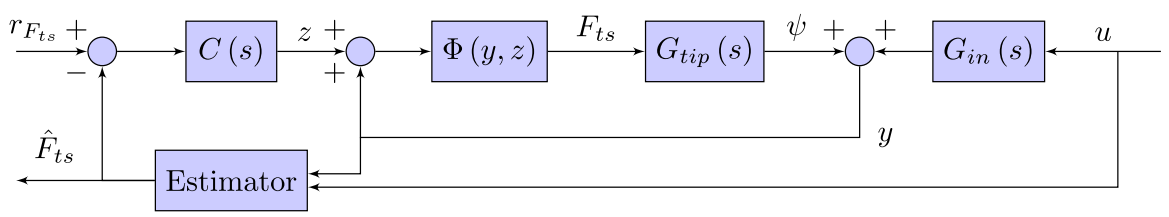

(b)

Fig. 3. Comparison of the conventional and proposed z-axis feedback control schemes (a) Conventional z-axis control scheme (b) Proposed z-axis control scheme.

where $T_{d}$ is chosen to capture the significant part of the impulse response. The tip-sample force $F_{\mathrm{ts}}(t)$ can then be expressed in terms of known quantities.

The problem can be further simplified with the assumption that the tip-sample force is an impulse train. This assumption is reasonable in tapping mode operation since the repulsive force component dominates the attractive component [24]. If the amplitude of oscillation of the microcantilever is large relative to the sample features, tip-sample contact is made at the same point in each oscillation cycle.

An impulse train or Dirac comb, with period $T$, can be mathematically expressed as

$$
I I I(t)=\sum_{k=-\infty}^{\infty} \delta(t-k T)
$$

where $\delta(t)$ denotes the Dirac delta function. The tip-sample force can then be defined as

$$
F_{\mathrm{ts}}(t)=\sum_{k=-\infty}^{\infty} a_{k} \delta(t-k T)
$$

where $a_{k}$ is the magnitude of the $k$ th impulse. Fig. 4 highlights the assumed form of the tip-sample force.

Given this structure of $F_{\mathrm{ts}}(t)$, using (4)

$$
\psi(t)=\sum_{k} a_{k} g_{\text {tip }}(t-k T)
$$

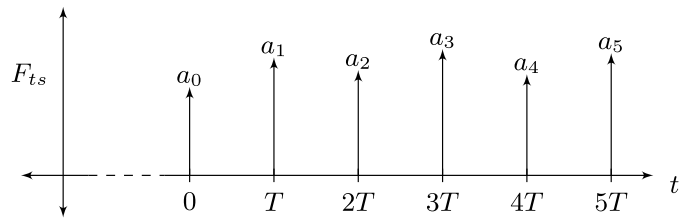

Fig. 4. In tapping mode AFM, the tip-sample force can be assumed to be an impulse train with period $T$.

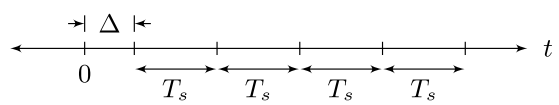

Fig. 5. Asynchronous sampling of the measured quantities at time $\Delta+\ell T_{s}$ with sampling period $T_{s}$.

where $a_{k}$ is the magnitude of the $k$ th impulse and $T$ is the period of the impulse train. It is assumed that the impulse response $g_{\text {tip }}(t)$ can be determined before execution. When tapping in air, the characteristically high-quality factor of the microcantilever means that the impulse response may require significant time to decay; force impulses in the past will contribute to the response of the microcantilever in the present. As an example, for a quality factor of 150 , it takes approximately 200 cycles for the impulse response to decay. It is important to highlight this for consideration of the real-time implementation. The technique becomes more computationally feasible when the impulse response decays rapidly, and use of this technique in liquid-based 


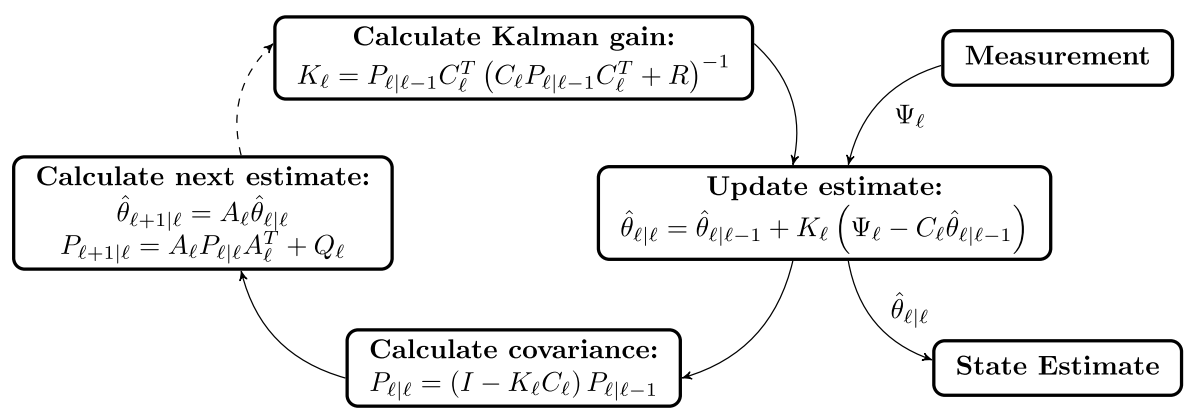

Fig. 6. Kalman filter equations for the tip-sample force estimation problem. Note that matrices $A_{l}$ and $Q_{l}$ will change during execution of the algorithm.

atomic force microscopy, where there is significant damping, may be possible [25].

Suppose $\psi(t)$ is asynchronously sampled with sampling pe$\operatorname{riod} T_{s}$ as shown in Fig. 5. Then define

$$
\begin{aligned}
& \Psi[\ell]=\psi\left(\ell T_{s}\right), \quad \forall \ell \in \mathbb{Z} \\
& g[\ell]=g_{\text {tip }}\left(\ell T_{s}\right), \quad \forall \ell \in \mathbb{Z}
\end{aligned}
$$

and from (8) it can be verified that

$$
\Psi[\ell]=\sum_{k=k_{1}}^{k_{2}} a_{k} g\left[\ell-k T / T_{s}\right]
$$

where

$$
\begin{aligned}
t(\ell) & =\Delta+\ell T_{s}, \quad \forall \ell \in \mathbb{Z} \\
k_{1}(\ell) & =\left\lfloor\frac{t(\ell)-\Delta}{T_{s}}\right\rfloor \\
k_{2}(\ell) & =\left\lceil\frac{t(\ell)-\Delta-T_{d}}{T_{s}}\right\rceil .
\end{aligned}
$$

It is important to recognize that due to the asynchronous sampling, the number of impulses whose responses must be considered in the convolution may not be constant. If the sampling rate were chosen to be an integer multiple of the resonance frequency (or the frequency of the Dirac comb), it would be possible to guarantee that there would always be a fixed number of impulses in this window; the state vector would have a fixed dimension. However, we outline the more general case here. The state vector in the $\ell$ th iteration $\theta_{\ell}$ can be defined as

$$
\theta_{\ell}=\left[\begin{array}{llll}
a_{k_{1}(\ell)} & a_{k_{1}(\ell)-1} & \cdots & a_{k_{2}(\ell)}
\end{array}\right]^{T} .
$$

At each sampling instant, the state vector must be reevaluated. In most instances, no new impulses will be present, however when a new impulse is expected, this vector must be updated.

\section{B. Kalman Filter Formulation}

The tip-sample force estimation problem can be formulated utilizing the Kalman filter framework. Consider the following discrete-time state space system:

$$
\begin{aligned}
\theta_{\ell+1} & =A_{\ell} \theta_{\ell}+\mu_{\ell} \\
\Psi_{\ell} & =C_{\ell} \theta_{\ell}+\nu_{\ell} .
\end{aligned}
$$

The system matrices are defined as

$$
\begin{aligned}
& \theta_{\ell}=\left[\begin{array}{llll}
a_{k_{1}(\ell)} & a_{k_{1}(\ell)-1} & \cdots & a_{k_{2}(\ell)}
\end{array}\right]^{T} \\
& C_{\ell}=\left[\begin{array}{c}
g_{\text {tip }}\left(t-k_{1}(\ell) T\right) \\
g_{\text {tip }}\left(t-\left(k_{1}(\ell)-1\right) T\right) \\
\vdots \\
g_{\text {tip }}\left(t-k_{2}(\ell) T\right)
\end{array}\right]^{T} \\
& \mu_{\ell}=\left\{\begin{array}{llll}
{\left[\begin{array}{llll}
0 & 0 & \cdots & 0
\end{array}\right]^{T},} & \text { no new impulse is detected } \\
{\left[\begin{array}{llll}
\delta & 0 & \cdots & 0
\end{array}\right]^{T},} & \text { a new impulse is detected }
\end{array}\right. \\
& \text { where } \delta \sim \mathcal{N}(0, \sigma) \\
& \nu_{\ell}=\mathcal{N}(0, \eta), R=\eta^{2} \text {. }
\end{aligned}
$$

As a result of the asynchronous sampling, the dimension $n$ of the system will likely vary. The system matrices must be modified accordingly. See Appendix A and B for details on updating the state transition matrix $A_{\ell}$ and the covariance matrix $Q_{\ell}$ during execution. When a new impulse is expected, the process noise $\mu_{\ell}$ is modified to highlight the uncertainty in the magnitude of this impulse; $\delta$ is a zero-mean, normally distributed random variable with standard deviation $\sigma$. While it is relatively straightforward to approximate $\eta$ from inspection of the measured data, several attempts may be necessary to determine a good value of $\sigma$. The Kalman filter equations are summarized in Fig. 6.

While an impulse is being tracked in the state vector, the Kalman filter will continue to refine the estimate of its magnitude based on new measurements. The best estimate of the mean amplitude of an impulse will be achieved after a $T_{d} \mathrm{~s}$ delay, however reliable estimates suitable for the feedback control can be produced in a much shorter timeframe.

Implementation of this estimation technique would then enable the regulation of the tip-sample force directly, instead of derivatives such as the amplitude. The tip-sample force estimates could be compared to a setpoint force as outlined in Fig. 3(b). Provided that the estimation technique converges rapidly, this control method will be superior to conventional $z$-axis control techniques, which are limited by the microcantilever dynamics and amplitude demodulation method. It is important to note that the estimator does require accurate knowledge of the impulse responses $g_{\text {in }}(t)$ and $g_{\text {tip }}(t)$. 


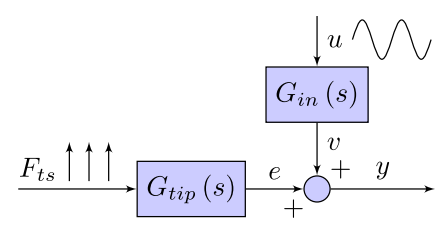

Fig. 7. During steady-state tapping, it is possible to estimate $g_{\text {tip }}(t)$.

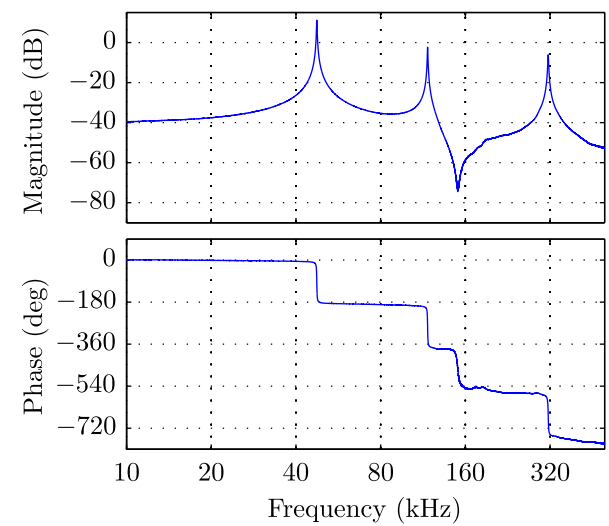

Fig. 8. Measured frequency response of $G_{\text {in }}(s)$.

\section{Identification of $G_{\mathrm{tip}}(s)$}

To determine $g_{\text {tip }}(t)$, we propose the following simple experiment. Since the system $G_{\text {in }}(s)$ is not affected by the nonlinear tip-sample force, it can be accurately characterized in free air. Fig. 8 highlights the first three modes of the identified model of $G_{\text {in }}(s)$. Once this model is known, we establish steady-state tapping of the microcantilever on a hard sample, such as a calibration grating, to generate a constant magnitude impulse train for the excitation of $G_{\text {tip }}(s)$ as shown in Fig. 7. With knowledge of $u(t), y(t)$, and $G_{\text {in }}(s)$, it is then possible to estimate $G_{\text {tip }}(s)$.

From Fig. 7 it can be seen that

$$
e(t)=y(t)-v(t)
$$

where $v(t)=\left(g_{\text {in }} * u\right)(t)$ and $y(t)$ is the measured tip displacement. Assuming steady-state operation and excitation with a fixed frequency $\omega_{0}$, then

$$
v(t)=G \cos \left(\omega_{0} t-\phi\right)
$$

where $G=\left\|G_{\text {in }}\left(\jmath \omega_{0}\right)\right\|$ and $\phi=\angle G_{\text {in }}\left(\jmath \omega_{0}\right)$.

Given the collocated nature of $G_{\text {tip }}(s)$ as shown in (2), $G_{\text {tip }}(s)$ can be expressed as the sum of second-order modes $M_{i}(s)$

$$
M_{i}(s)=\frac{\gamma_{i} \omega_{i}^{2}}{s^{2}+2 \zeta_{i} \omega_{i} s+\omega_{i}^{2}}, \gamma_{i}>0 .
$$

The impulse response $g_{\text {tip }}(t)$ can be determined by taking the inverse Laplace transform of $G_{\text {tip }}(s)$. Let us rewrite $M_{i}(s)$ as

$$
M_{i}(s)=\frac{\gamma_{i} \omega_{i}^{2}}{s^{2}+2 \zeta_{i} \omega_{i} s+\omega_{i}^{2}}
$$

$$
=\frac{\gamma_{i} \omega_{i}}{\sqrt{1-\zeta_{i}^{2}}} \frac{\sqrt{1-\zeta_{i}^{2}} \omega_{i}}{\left(s+\zeta_{i} \omega_{i}\right)^{2}+\left(1-\zeta_{i}^{2}\right) \omega_{i}^{2}} .
$$

It is straightforward to determine the inverse Laplace transform as

$$
\begin{aligned}
m_{i}(t) & =\frac{\gamma_{i} \omega_{i}}{\sqrt{1-\zeta_{i}^{2}}} e^{-\zeta_{i} \omega_{i} t} \sin \left(\sqrt{1-\zeta_{i}^{2}} \omega_{i} t\right) \\
& =c_{i} e^{-\alpha_{i} t} \sin \left(\beta_{i} t\right)
\end{aligned}
$$

where

$$
\begin{aligned}
c_{i} & =\frac{\gamma_{i} \omega_{i}}{\sqrt{1-\zeta_{i}^{2}}} \\
\alpha_{i} & =\zeta_{i} \omega_{i} \\
\beta_{i} & =\sqrt{1-\zeta_{i}^{2}} \omega_{i} .
\end{aligned}
$$

However, $-\alpha_{i} \pm \jmath \beta_{i}$ are known from the identification of $G_{\text {in }}(s)$. Only the coefficients $c_{i}$ remain to be determined. The linearity property of the Laplace transform allows us to determine the impulse response $m_{i}(t)$ for each mode $M_{i}(s)$ and to sum these together to determine the overall impulse response $g_{\text {tip }}(t)$. If $N$ is the number of modes under consideration, then

$$
g_{\text {tip }}(t)=\sum_{i=1}^{N} c_{i} e^{-\alpha_{i} t} \sin \left(\beta_{i} t\right) .
$$

During steady-state tapping, the measured displacement signal is periodic and a least squares estimate of the impulse response can be formulated utilizing only a single period of the signal. For each sampled point in time $0<\tau<T$, the following relationship exists:

$$
e(\tau)=\left[\begin{array}{c}
1 \\
\sum_{k=0}^{\infty} e^{-\alpha_{1}(\tau+k T)} \sin \left(\beta_{1}(\tau+k T)\right) \\
\sum_{k=0}^{\infty} e^{-\alpha_{2}(\tau+k T)} \sin \left(\beta_{2}(\tau+k T)\right) \\
\vdots \\
\sum_{k=0}^{\infty} e^{-\alpha_{N}(\tau+k T)} \sin \left(\beta_{N}(\tau+k T)\right)
\end{array}\right]^{T}\left[\begin{array}{c}
c_{0} \\
c_{1} \\
c_{2} \\
\vdots \\
c_{N}
\end{array}\right] .
$$

The least squares estimate of the coefficients $c_{i}$ then forms the estimate for $g_{\text {tip }}(t)$. The transfer function $G_{\text {tip }}(s)$ can also be determined. The coefficient $c_{0}$ is present to account for the dc component in $e(t)$.

Measured data were obtained to estimate $g_{\text {tip }}(t)$. An NTMDT Ntegra AFM and a Bruker DMASP microcantilever were utilized. To ensure strong tip-sample interactions, lowamplitude setpoints were utilized. The signals $u(t)$ and $y(t)$ were recorded using an Agilent DSO90254A oscilloscope sampling at $20 \mathrm{MHz}$. The measured displacement signal $y(t)$ is shown in Fig. 11; the significant distortion in the waveform is the result of the strong tip-sample interaction forces. An accurate model of $G_{\text {in }}(s)$ was obtained using a Zurich Instruments HF2LI lock-in amplifier; the quality factors were measured for each mode to determine $\alpha_{i} \pm \jmath \beta_{i}$.

The proposed identification technique requires that the force impulse occurs at $t=0$. Fig. 12 shows one period of the 

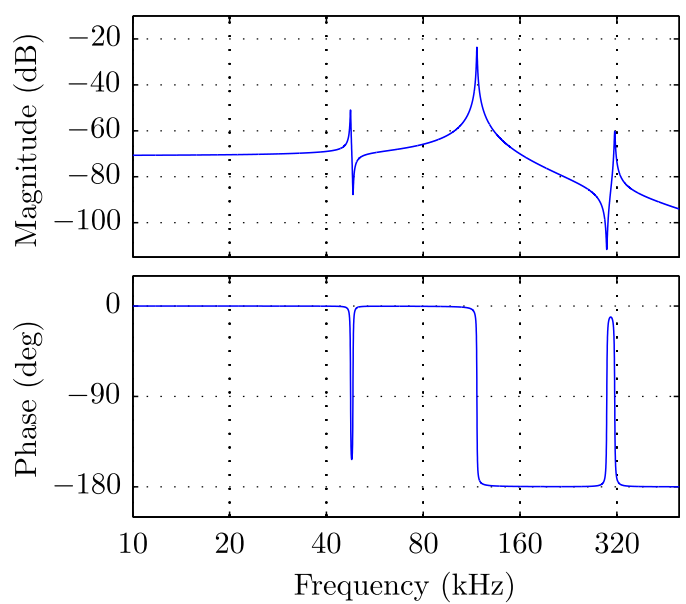

Fig. 9. Estimated frequency response of $G_{\text {tip }}(s)$.

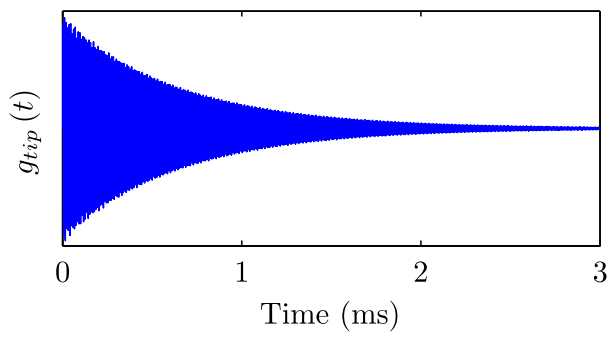

Fig. 10. Estimated impulse response $g_{\text {tip }}(t)$.

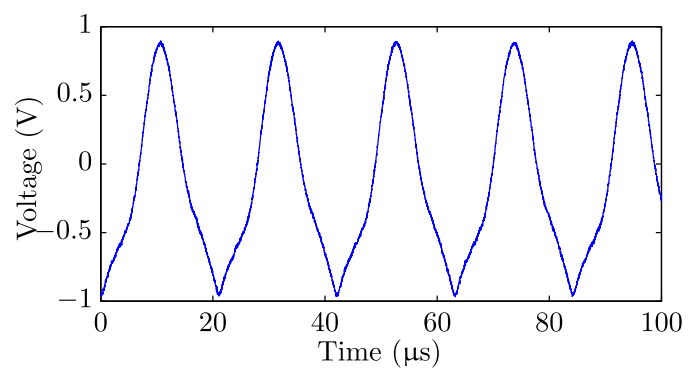

Fig. 11. Measured displacement signal $y(t)$; tip-sample contact occurs at the lowest part of the cycle. The strong tip-sample forces are responsible for the significant distortion.

measured tip displacement, $e(t)$ and $\bar{e}(t)$, which was calculated using the estimate of $g_{\text {tip }}(t)$. Clearly there is very good agreement between $e(t)$ and $\bar{e}(t)$. The validation data, which were obtained from a separate experiment utilizing a lower setpoint amplitude, was used to confirm the accuracy of $g_{\text {tip }}(t)$. The impulse response $g_{\text {tip }}(t)$ was identified as shown in Fig. 10. The duration of the impulse response $T_{d}$ was chosen to be $3 \mathrm{~ms}$, which corresponds to the time it takes for the impulse response to decay to $1 \%$ of its original value. However, $T_{d}$ is a design parameter and can be determined by the user. Decreasing $T_{d}$ reduces the computational requirements of the estimation technique since fewer impulses must be tracked and estimated in the Kalman filter, but may result in a loss of accuracy since the
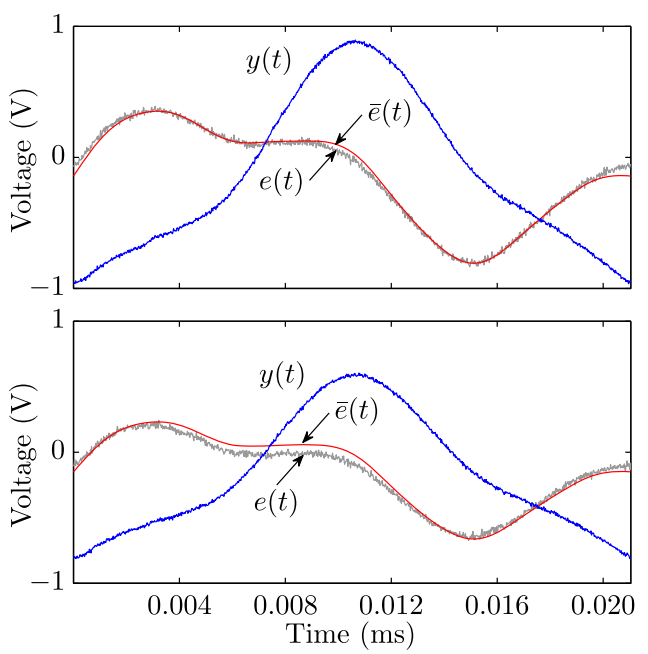

Fig. 12. One period of the measured displacement signal (-), $e(t)(-)$, and $\bar{e}(t)$, which was calculated using the estimate of $g_{\mathrm{tip}}(t)(-)$ (top) and validation of the estimated $g_{\text {tip }}(t)$ with new data.

algorithm is not accounting for all of the tip-sample interactions in the past.

Interestingly, it was discovered that the second mode dominates the impulse response; the first mode is not particularly significant. This observation is consistent with experimentation on larger cantilevers [23]. The frequency responses of $G_{\text {in }}(s)$ and $G_{\text {tip }}(s)$ are shown in Figs. 8 and 9, respectively. While the poles are identical, clearly the input-output relationships are very different and given the discussion in Section III, this is not surprising.

\section{Offline Analysis}

To test the effectiveness of the proposed technique, experimental data were obtained. Using the same experimental setup, images of an NT-MDT TGZ1 calibration grating, featuring step heights of $21.6 \pm 1.5 \mathrm{~nm}$, were obtained. The amplitude setpoint was $50 \%$ and the actuation voltage was $0.1 \mathrm{~V}$. The standard $z$ axis controller was utilized; the intention here is to highlight operation of the tip-sample force estimation technique using experimental data. The signals $u(t)$ and $y(t)$ were recorded using an Agilent DSO90254A oscilloscope sampling at $20 \mathrm{MHz}$. The sample topography and error signals were recorded to verify that the features in the tip-sample force estimate were synchronized with the sample features. The estimation technique was implemented in MATLAB.

The estimated tip-sample force is shown in Fig. 13. The tipsample force estimate is compared with the measured sample topography (z-axis controller output) and error signal. While it is not possible to compare the estimated tip-sample force $\hat{F}_{\mathrm{ts}}$ with the actual tip-sample force, the force estimate clearly reflects the sudden changes in the calibration grating, which is a promising sign for high-bandwidth control and agrees with AFM simulations. Fig. 14 provides a close-up view of the transients in the estimated tip-sample force. 

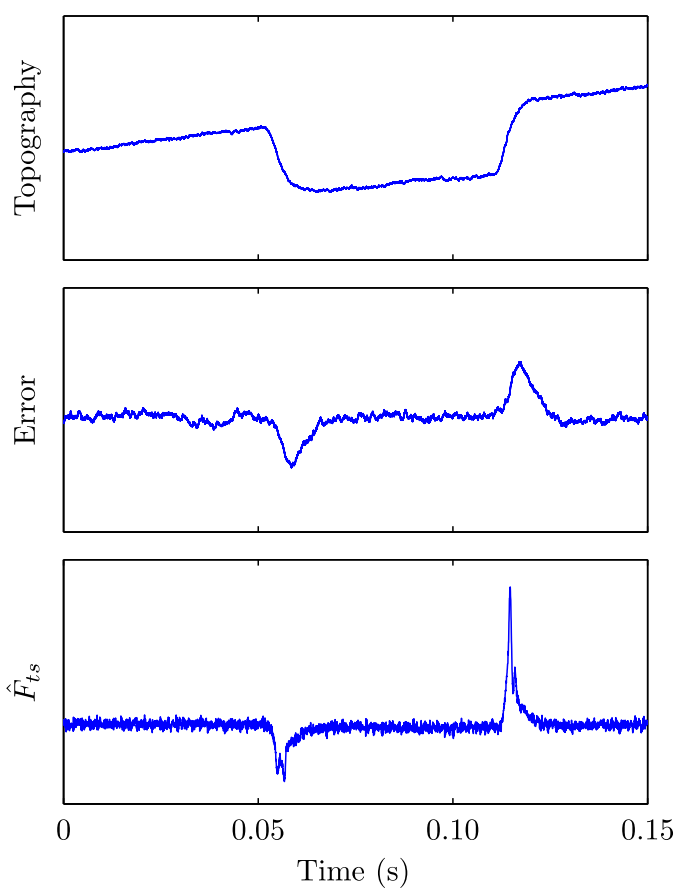

Fig. 13. Measured sample topography (top), amplitude error signal (middle), and tip-sample force estimate $\hat{F_{\mathrm{ts}}}$ (bottom). Using the proposed tip-sample force estimation technique, both upward and downward steps are clearly visible in the force estimate.

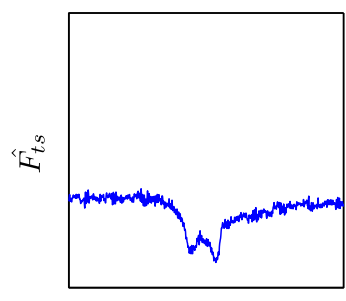

Time

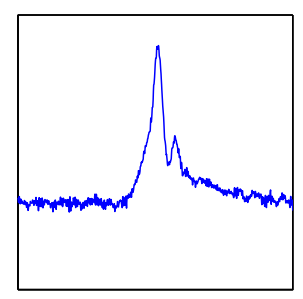

Time
Fig. 14. Close-up view of the tip-sample force estimate highlighting transients in the force when a sample feature is encountered.

At this stage, we do not determine the magnitude of the tipsample force; this would require an additional calibration step, which is currently under investigation. We also believe that issues such as sensor loss, which can be easily identified in the proposed scheme since there would be no tip-sample interaction, can be easily addressed similar to the method proposed in [26]. Given the high-bandwidth nature of the technique, high-speed control could be implemented to ensure that the cantilever is quickly recoupled with the sample.

We believe that high-bandwidth $z$-axis control could be implemented utilizing accurate models of the microcantilever. One significant advantage of this approach is that cantilevers are less prone to change over time and do not suffer as noticeably from hysteresis and creep [27] as do the majority of $z$-axis nanopositioners. Furthermore, the bandwidth of the microcantilever is significantly higher than that of the $z$-axis nanopositioner. This technique successfully eliminates the dependence of the $z$-axis control loop bandwidth on the transient response of the microcantilever; instead, it utilizes the high-frequency information in the displacement signal. Increasing the bandwidth of the $z$ axis control loop enables the loop gain to be increased while maintaining stability.

While the experimental verification only considered actuation of the microcantilever at a single frequency, the technique can be extended for compatibility with multifrequency excitation. Consider multiple excitation frequencies in $u(t)$, then

$$
v(t)=\sum_{i=1}^{N} G_{i} \cos \left(\omega_{i} t-\phi_{i}\right)
$$

where $G_{i}=\left\|G_{\text {in }}\left(\jmath \omega_{i}\right)\right\|$ and $\phi_{i}=\angle G_{\text {in }}\left(\jmath \omega_{i}\right)$. Once $e(t)$ has been calculated, the Kalman filter can be implemented. However, this is only possible if the tip-sample force can be accurately modeled as a uniformly spaced impulse train.

\section{CONCLUSION}

We have provided new insights into the modeling of the microcantilever in dynamic mode atomic force microscopy and outlined a novel tip-sample estimation technique proposed specifically to improve the bandwidth of the $z$-axis control loop, thus resulting in improved imaging bandwidth. Assuming that the tip-sample force takes the form of an impulse train, the estimation problem can be formulated as a Kalman filter. A novel identification technique for determining $G_{\text {tip }}(s)$ was also proposed. Measured data were used for experimental verification; the estimated tip-sample force $\hat{F}_{\mathrm{ts}}$ may be useful for highbandwidth control purposes. The next step in the development of high-bandwidth $z$-axis control is a high-speed implementation on an FPGA.

\section{APPENDIX A}

UPDATING THE State TRANSITION Matrix $A_{l}$

Recall that $t(\ell)=\Delta+\ell T_{s} \quad \forall \ell \in \mathbb{Z}$, therefore

$$
\begin{aligned}
& k_{1}(\ell)=\left\lfloor\frac{t(\ell)-\Delta}{T_{s}}\right\rfloor \\
& k_{2}(\ell)=\left\lceil\frac{t(\ell)-\Delta-T_{d}}{T_{s}}\right\rceil .
\end{aligned}
$$

The dimension $n$ of the system in each iteration is

$$
n(\ell)=k_{1}(\ell)-k_{2}(\ell)+1 .
$$

Knowledge of $n(\ell), n(\ell-1), k_{1}(\ell), k_{1}(\ell-1), k_{2}(\ell)$, and $k_{2}(\ell-1)$ are sufficient to determine when the state transition matrix must be modified.

Consider the four possible scenarios. 
1) If $k_{1}(\ell)=k_{1}(\ell-1)$, and $k_{2}(\ell)=k_{2}(\ell-1)$, there is no change in the state vector $\theta_{\ell}$ and $A_{\ell}$ is the identity matrix

$$
A_{\ell}=\left[\begin{array}{ccccc}
1 & 0 & 0 & \cdots & 0 \\
0 & 1 & 0 & \cdots & 0 \\
0 & 0 & 1 & \cdots & 0 \\
\vdots & \vdots & \vdots & \ddots & \vdots \\
0 & 0 & 0 & \cdots & 1
\end{array}\right]_{n \times n} .
$$

2) If $n(\ell)=n(\ell-1)$ but $k_{1}$ and $k_{2}$ both change, then the number of impulses in the state vector remains constant. $A_{\ell}$ remains square, but is defined as

$$
A_{\ell}=\left[\begin{array}{ccccc}
1 & 0 & \cdots & 0 & 0 \\
1 & 0 & \cdots & 0 & 0 \\
0 & 1 & \cdots & 0 & 0 \\
\vdots & \vdots & \ddots & & \vdots \\
0 & 0 & \cdots & 1 & 0
\end{array}\right]_{n \times n}
$$

where the mean amplitude of the new impulse $a_{0}$ is assumed to be identical in magnitude to the most recent impulse $a_{1}$.

3) If $n(\ell)>n(\ell-1)$, then a new impulse is expected in the state vector. The dimension of the system will change and $A_{\ell}$ is nonsquare. It is assumed that the new impulse is identical in magnitude to the most recent one.

$$
A_{\ell}=\left[\begin{array}{ccccc}
1 & 0 & 0 & \cdots & 0 \\
1 & 0 & 0 & \cdots & 0 \\
0 & 1 & 0 & \cdots & 0 \\
0 & 0 & 1 & \vdots & 0 \\
\vdots & \vdots & \vdots & \ddots & \vdots \\
0 & 0 & 0 & \cdots & 1
\end{array}\right]_{(n+1) \times n}
$$

4) If $n(\ell)<n(\ell-1)$, then an old impulse has disappeared from the state vector. The dimension of the system will change and $A_{\ell}$ is nonsquare.

$$
A_{\ell}=\left[\begin{array}{ccccc}
1 & 0 & \cdots & 0 & 0 \\
0 & 1 & \cdots & 0 & 0 \\
\vdots & \vdots & \ddots & & \vdots \\
0 & 0 & 0 & 1 & 0
\end{array}\right]_{(n-1) \times n}
$$

\section{APPENDIX B}

\section{Predicting the Covariance Matrix $P_{\ell+1 \mid \ell}$}

The process noise $\mu_{\ell}$ is only present when a new impulse is expected. The estimate of the covariance matrix $P_{\ell}$ is determined by

$$
P_{\ell+1 \mid \ell}=A_{\ell} P_{\ell \mid \ell} A_{\ell}^{T}+Q_{\ell}
$$

The $Q_{\ell}$ matrix is representative of the uncertainty in the magnitude of the new impulse. The covariance matrix $Q_{\ell}$ may be defined as follows.

(1) When there are no new impulses expected $Q_{\ell}=[0]_{n \times n}$.

(2) When a new impulse is expected

$$
Q_{\ell}=\left[\begin{array}{ccc}
\sigma^{2} & \cdots & 0 \\
\vdots & \ddots & \vdots \\
0 & \cdots & 0
\end{array}\right]_{n \times n} .
$$

\section{REFERENCES}

[1] G. Binnig, C. F. Quate, and C. Gerber, "Atomic force microscope," Phys. Rev. Lett., vol. 56, no. 9, pp. 930-933, 1986.

[2] Y. Martin, C. C. Williams, and H. K. Wickramasinghe, "Atomic force microscope-force mapping and profiling on a sub 100-Åscale," J. Appl. Phys., vol. 61, no. 10, pp. 4723-4729, 1987.

[3] Q.Zhong, D. Inniss, K. Kjoller, and V. B. Elings, "Fractured polymer/silica fiber surface studied by tapping mode atomic force microscopy," Surf. Sci., vol. 290, nos. 1/2, pp. L688-L692, 1993.

[4] T. Ando, "High-speed atomic force microscopy coming of age," Nanotechnology, vol. 23, no. 6, pp. 062001-1-062001-27, 2012.

[5] A. Sebastian, M. V. Salapaka, and J. P. Cleveland, "Robust control approach to atomic force microscopy," in Proc. IEEE Conf. Decision Control, 2003, vol. 4, pp. 3443-3444.

[6] S. M. Salapaka, T. De, and A. Sebastian, "A robust control based solution to the sample-profile estimation problem in fast atomic force microscopy," Int. J. Robust Nonlinear Control, vol. 15, no. 16, pp. 821-837, 2005.

[7] G. Schitter and N. Phan, "Field programmable analog array (FPAA) based control of an atomic force microscope," in Proc. Amer. Control Conf., 2008, pp. 2690-2695.

[8] K. S. Karvinen and S. O. R. Moheimani. (2014). Modulateddemodulated control: Q control of an AFM microcantilever. Mechatronics [Online]. vol. 24, no. 6, pp. 661-671. Available: http://www.sciencedirect.com/science/article/pii/S0957415813002316

[9] M. W. Fairbairn, S. O. R. Moheimani, and A. J. Fleming, " $Q$ control of an atomic force microscope microcantilever: A sensorless approach," J. Microelectromech. Syst., vol. 20, no. 6, pp. 1372-1381, 2011.

[10] M. W. Fairbairn and S. O. R. Moheimani, "Control techniques for increasing the scan speed and minimizing image artifacts in tapping mode atomic force microscopy: Toward video-rate nanoscale imaging," IEEE Control Syst. Mag., vol. 33, no. 6, pp. 46-67, Dec. 2013.

[11] K. S. Karvinen and S. O. R. Moheimani, "A high-bandwidth amplitude estimation technique for dynamic mode atomic force microscopy," Rev. Sci. Instrum., vol. 85, no. 2, p. 023707, 2014.

[12] N. Kodera, D. Yamamoto, R. Ishikawa, and T. Ando, "Video imaging of walking myosin V by high-speed atomic force microscopy," Nature, vol. 468, no. 7320, pp. 72-76, 2010.

[13] M. Shibata, H. Yamashita, T. Uchihashi, H. Kandori, and T. Ando, "Highspeed atomic force microscopy shows dynamic molecular processes in photoactivated bacteriorhodopsin," Nature Nanotechnol., vol. 5, no. 3, pp. 208-212, 2010.

[14] T. Uchihashi, R. Iino, T. Ando, and H. Noji, "High-speed atomic force microscopy reveals rotary catalysis of rotorless $\mathrm{F}_{1}$-ATPase," Science, vol. 333, no. 6043, pp. 755-758, 2011.

[15] S. Necipoglu, S. Cebeci, Y. Has, L. Guvenc, and C. Basdogan, "Robust repetitive controller for fast AFM imaging," IEEE Trans. Nanotechnol., vol. 10, no. 5, pp. 1074-1082, Sep. 2011.

[16] Y. Jeong, G. R. Jayanth, S. M. Jhiang, and C. Menq, "Direct tip-sample interaction force control for the dynamic mode atomic force microscopy," Appl. Phys. Lett., vol. 88, no. 20, pp. 204102-1-204102-3, 2006.

[17] D. R. Sahoo, A. Sebastian, and M. V. Salapaka, "Transient-signal-based sample-detection in atomic force microscopy," Appl. Phys. Lett., vol. 83, no. 26, pp. 5521-5523, 2003.

[18] D. R. Sahoo, A. Sebastian, and M. V. Salapaka, "Harnessing the transient signals in atomic force microscopy," Int. J. Robust Nonlinear Control, vol. 15 , pp. 805-820, 2005.

[19] A. Sebastian, M. V. Salapaka, D. J. Chen, and J. P. Cleveland, "Harmonic and power balance tools for tapping-mode atomic force microscope," J. Appl. Phys., vol. 89, no. 11, pp. 6473-6480, 2001. 
[20] R. W. Stark, G. Schitter, M. Stark, R. Guckenberger, and A. Stemmer, "State-space model of freely vibrating and surface-coupled cantilever dynamics in atomic force microscopy," Phys. Rev. B, Condens. Matter Mater. Phys., vol. 69, no. 8, pp. 854121-854129, 2004.

[21] A. Sebastian, A. Gannepalli, and M. V. Salapaka, "A review of the systems approach to the analysis of dynamic-mode atomic force microscopy," IEEE Trans. Control Syst. Technol., vol. 15, no. 5, pp. 952-959, Sep. 2007.

[22] D. Halim and S. O. R. Moheimani, "Reducing the effect of truncation error in spatial and pointwise models of resonant systems with damping," Mech. Syst. Signal Process., vol. 18, no. 2, pp. 291-315, 2004.

[23] S. O. R. Moheimani and A. J. Fleming, Piezoelectric Transducers for Vibration Control and Damping (Advances in Industrial Control Series). New York, NY, USA: Springer, 2006.

[24] R. García and A. San Paulo, "Attractive and repulsive tip-sample interaction regimes in tapping-mode atomic force microscopy," Phys. Rev. B, vol. 60, pp. 4961-4967, Aug. 1999.

[25] T. E. Schäffer, J. P. Cleveland, F. Ohnesorge, D. A. Walters, and P. K. Hansma, "Studies of vibrating atomic force microscope cantilevers in liquid," J. Appl. Phys., vol. 80, no. 7, pp. 3622-3627, 1996.

[26] P. Agarwal, T. De, and M. V. Salapaka, "Real time reduction of probe-loss using switching gain controller for high speed atomic force microscopy," Rev. Sci. Instrum., vol. 80, no. 10, p. 103701, 2009.

[27] S. Devasia, E. Eleftheriou, and S. O. R. Moheimani, "A survey of control issues in nanopositioning," IEEE Trans. Control Syst. Technol., vol. 15, no. 5, pp. 802-823, Sep. 2007.

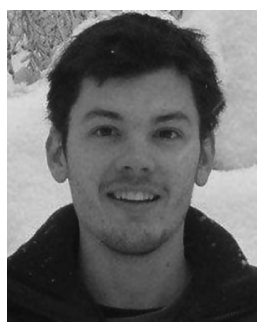

Kai S. Karvinen (S'14) graduated with a double degree in electrical engineering and applied physics from Curtin University, Bentley, WA, Australia, in 2009. He received the Ph.D. degree in electrical engineering from The University of Newcastle, Callaghan, NSW, Australia, in 2014.

His research interests include the development of control and estimation techniques for high-bandwidth dynamic mode atomic force microscopy.

Dr. Karvinen received the Digby Leach Medal, the Engineers Australia Electrical College Bruce James Medal, and the Physics Graduate Award for his outstanding academic achievements.

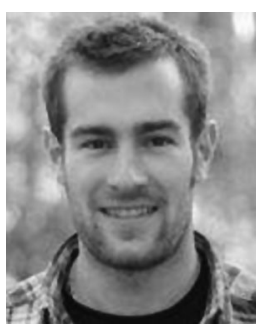

Michael G. Ruppert (S'14) received the Dipl.Ing. degree in automation technology in production with a specialization in systems theory and automatic control from the University of Stuttgart, Stuttgart, Germany, in 2013. He is currently working toward the $\mathrm{Ph} . \mathrm{D}$. degree in electrical engineering from The University of Newcastle, Callaghan, NSW, Australia.

His research interests include the application of systems theory to multifrequency atomic force microscopy and multimodal $Q$-control of cantilevers.

Mr. Ruppert received an academic merit scholarship and the Baden-Württemberg scholarship.

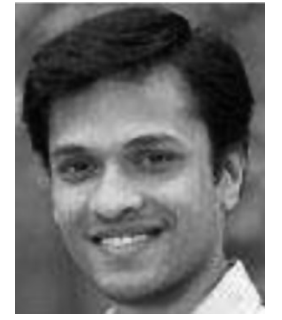

Kaushik Mahata received the Ph.D. degree in signal processing from Uppsala University, Uppsala, Sweden, in 2003.

Since 2003, he has been with the University of Newcastle, Callaghan, NSW, Australia, where he is currently an Associate Professor. His research interests include system identification, spectral analysis, compressive sensing, and telecommunication networks.

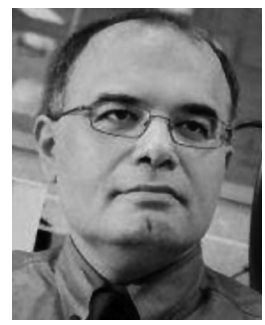

S. O. R. Moheimani ( $\left.F^{\prime} 11\right)$ received the Bachelor's degree in electrical engineering from Shiraz University, Shiraz, Iran, in 1991, and the Ph.D. degree from the University of New South Wales, Sydney NSW, Australia, in 1996.

He joined the University of Newcastle in 1997, embarking on a new research program addressing the dynamics and control design issues related to highprecision mechatronic systems. He is the Founder and Director of the Laboratory for Dynamics and Control of Nanosystems, a multimillion-dollar state-of-theart research facility. He has published more than 300 refereed papers and five books and edited volumes. His current research interests include ultrahighprecision mechatronic systems, with particular emphasis on dynamics and control at the nanometer scale, including applications of control and estimation in nanopositioning systems for high-speed scanning probe microscopy, modeling and control of microcantilever-based devices, control of micro-actuators in microelectromechanical systems, and design, modeling and control of micromachined nanopositioners for on-chip atomic force microscopy.

Dr. Moheimani is a Fellow of the International Federation of Automatic Control (IFAC) and the Institute of Physics, U.K. His work has been recognized with a number of awards, including the IFAC Nathaniel B. Nichols Medal in 2014, the IFAC Mechatronic Systems Award in 2013, the IEEE Control Systems Technology Award in 2009, the Australian Research Council Future Fellowship in 2009, the IEEE TRAnsaCtions on ConTrol Systems TeChNOLOGY Outstanding Paper Award in 2007, and the Australian Research Council Postdoctoral Fellowship in 1999. He has served on the Editorial Boards of a number of journals, including the IEEE/ASME TRANSACTIONS ON MECHATRONICS, the IEEE TRANSACtions on Control Systems TeChNOlogy, and Control Engineer ing Practice. He has chaired several international conferences and workshops, and he is currently a chair of the IFAC Technical Committee on Mechatronic Systems. 